\title{
Entrelacs
}

Cinéma et audiovisuel

$10 \mid 2013$

Le Toucher

\section{L'image-coupable : haptique du regard dans le cinéma de Larry Clark}

\section{Anna Muslewski}

\section{(2) OpenEdition}

\section{Journals}

Édition électronique

URL : http://journals.openedition.org/entrelacs/526

DOI : 10.4000/entrelacs.526

ISSN : 2261-5482

Éditeur

Éditions Téraèdre

Référence électronique

Anna Muslewski, «L'image-coupable : haptique du regard dans le cinéma de Larry Clark », Entrelacs [En ligne], 10 | 2013, mis en ligne le 12 septembre 2013, consulté le 30 avril 2019. URL : http:// journals.openedition.org/entrelacs/526; DOI : 10.4000/entrelacs.526

Ce document a été généré automatiquement le 30 avril 2019.

Tous droits réservés 


\title{
L'image-coupable : haptique du regard dans le cinéma de Larry Clark
}

\author{
Anna Muslewski
}

1 Le cinéma de Larry Clark est un art éminemment tactile. Sa caméra, focalisée sur les corps meurtris de l'adolescence américaine marginale, s'abandonne éperdument, compulsivement, sur ses kids à peine teens, consumés par la drogue et l'excès, brutalisés par leurs pairs et leurs pères, fourvoyés dans la violence et la criminalité. Collée à leur peau, elle façonne une matérialité organique au corps filmique, une corporéité, qui réifiée par la mise en image de la chair sexuée, se fonde sur un rapport exalté du toucher. La proximité, le contact, la palpation, sont en effet autant de manifestations sensibles jouant un rôle prépondérant dans la structure de l'image cinématographique, tant d'un point de vue narratif (dans la diégèse, la relation des personnages entre eux s'effectuent par attouchement) que formel (le filmage, notamment en mode frontal et en plans rapprochés, favorise l'extension des sens) et perceptif (la représentation iconographique des corps participe d'une préhension haptique et kinesthésique du medium).

2 Incarnant ce que d'ordinaire le cinéma refoule dans l'intimité de son hors-champ, l'image clarkienne manifeste continûment les obsessions de son créateur: un désir de «tout montrer », une exigence d'être " au plus près » de la réalité filmée, une volonté de rendre visible l'invisible. De fait, intime et sexuée, elle est d'emblée condamnée à devoir se justifier, en ce sens où, corporéisée, elle déborde de visibilité et reste toujours empreinte de culpabilité. Présentifiée par un cadrage suggestif qui dénude les corps à l'image, par une caméra baladeuse qui effleure leur surface, par des plans caressant les chairs et souillant les regards, cette culpabilité participe à corrompre l'image en invitant à l'infiltrer par contact. De la proximité visuelle à la frontalité de la mise en scène, l'esthétique clarkienne élabore donc une autre expérience du regard qui, substituant la dimension optique de la perception visuelle à une dimension haptique ${ }^{3}$, s'exprime par une sensorialité optico-tactile ${ }^{4}$. Ce texte se propose d'en réfléchir les modalités sensibles. 


\section{Prise de contact}

3 L'adolescence dans le cinéma de Clark, et dans son œuvre de manière générale, est une affaire de corps. Corps délabrés, tourmentés, contaminés qu'une caméra attentionnée sublime, mais aussi corps convoités, désirés ou répugnés qu'une œillade inquisitrice voudrait consommer. Au fond, la figuration des corps, préservés par la sollicitude d'autrui ou asservis à sa perversité, scinde en une partition tendancielle la double modalité du regard que porte l'adulte sur la jeunesse et ses images. Un regard ambigu qui, désirant voir et prendre contact, trahit sa concupiscence quand il n'exprime pas ses intentions malfaisantes. Rares sont en effet les représentations positives de l'adulte et de la parentalité. On ne les décèle à vrai dire que dans Wassup Rockers (2005) à travers la figure protectrice de la mère et celle de la communauté latine de South Central. Soumis à un traitement vitriolé, ces "portraits crachés $\|^{5}$, pour reprendre les mots de Mia HansenLove, demeurent constamment entachés de monstruosité.

4 L'adulte, défaillant, et qui se refuse en tant que tel, est toujours un principe nocif, un élément toxique : soit il est démissionnaire (par son absence ou son indifférence) soit il règne en tortionnaire (l'adolescent chez Clark est sans cesse en proie aux agressions parentales, qu'elles soient physiques, verbales ou sexuelles). Les parents de Kids (1995) sont inexistants ; effacés du monde de leurs progénitures, ils résident, invisibles, dans un hors-champ silencieux. Ceux d'Another Day in Paradise (1998) sont de substitution. Gangsters aguerris, ils incarnent la figure tutélaire en adoptant, le temps d'un braquage et de quelques shoots d'héroïne, un couple d'adolescents à la dérive. Dans Bully (2001), l'aveuglement, le désistement et l'incompréhension des adultes responsables constituent la cause du mal-être des teenagers et, indirectement, l'origine de leur comportement barbare, lequel les conduit à orchestrer un massacre sauvage ${ }^{6}$. Alors que dans Teenage Caveman (2002), l'expression de l'autorité parentale passe par un pouvoir patriarcal ${ }^{7}$, celle de Ken Park (2002), dont on retrouve le substrat symbolique du précédent téléfilm ${ }^{8}$, est soumise à l'instinct pervers d'une sexualité incestueuse. ${ }^{9}$

5 Si la relation des adolescents aux adultes est malaisée ${ }^{10}$ - leur contact ne peut être qu'hostile et torturé - celle des teens entre eux n'est guère plus sereine, exception faite du septuor punk rock et fraternel des skateurs-baroudeurs de Wassup Rockers, ainsi que du trio complice qui clôt sexuellement la fin du sulfureux Ken Park. Conditionné à la spontanéité de l'instinct et aux passions, le rapport au corps de l'autre est construit sur une attraction physique exaspérée. Une force, en somme, irrépressible, qui gouverne les relations charnelles et les consume fâcheusement (infection, viol, meurtre), mais qui, au-delà de toute détermination narrative, manifeste un besoin d'appropriation du corps désiré, une envie de le chosifier. Et cette dynamique contrariée, véritable "captativité »"11, au demeurant assez proche de celle caractérisant le comportement suspect des parents, définit l'expérience de la chair à laquelle chaque adolescent se livre ou est livré, soit une relation fondée sur la pénétration des corps et leur dégradation.

6 Dans Kids, Telly, "accro aux vierges » selon ses termes, excelle dans la conquête et la domestication des jeunes filles. Littéralement obnubilé par sa quête de pureté, il les séduit sans grande difficulté et finit toujours par les déflorer. Mais si la défloration est en soi une altération $^{12}$, la dégradation des corps concerne ici la contamination à laquelle ils sont soumis. Telly est en effet atteint du virus du sida. Bien qu'il ignore sa maladie, chacune de ses liaisons est néanmoins une contagion, et son infection, propagée à l'insu des corps 
jouissants, parfois dans la douleur mais toujours par consentement, se transmet aussi par violation (le viol de Jenny, séropositive, abusée durant son sommeil par Casper). Dans Bully, le contact des corps s'établit également par la violence et se réalise par l'intermédiaire de la souffrance et du châtiment. Le supplice y est norme. Bobby martyrise Marty son meilleur ami. Il l'humilie, le moleste, l'asservit. Sa relation aux autres est perverse et oppressive. Il maltraite ses partenaires sexuelles (il viole Ali après l'avoir forcée à visionner une vidéo pornographique), méprise son entourage (sauf sa famille devant laquelle, face à son père, il s'incline) et répugne systématiquement le corps d'autrui (il frappe, fustige et insulte ses amis). La particularité de cette animosité, outre son insupportable gratuité, vient du fait qu'instinctivement, elle prend forme dans l'ensemble de la communauté et, s'emparant un à un des adolescents, elle s'impose naturellement comme la seule connexion possible, le seul moyen de communiquer entre les corps. Et, dans ce corps à corps barbare, l'ultime pénitence, le meurtre du bourreau, apparait comme une évidence. Une évidence qui se profilait déjà dans Kids (la scène de rixe où la horde d'adolescents déchaînés menée par Casper s'acharne violemment sur un autre jeune) et qui se délitera dans Wassup Rockers (les skateurs ne cesseront tout au long de leur périple d'esquiver la violence des « autres " à leur égard). Si le contact entre les corps dans la diégèse traduit toujours, in fine, un rapport à la chair trouble et complexé, il est intéressant de s'interroger sur la relation qui s'instaure entre le corps regardant, celui du spectateur et celui du filmeur, et le corps regardé, celui de l'image.

\section{Filmer à corps perdu}

7 En accord avec sa démarche de visibilité - tout dire, tout voir, tout montrer - la représentation des corps chez Clark, inévitablement, passe par une mise en image de la chair sexuée. Refusant toute concession quant au sujet traité - l'univers tourmenté, intime et caché des kids - et obéissant à son obsession de dé-voiler (de toucher la réalité), le cinéaste place sa caméra in media res et n'hésite pas à révéler ce que communément d'aucuns se refuse à filmer, le sexe teen. Ce traitement particulier accordé à la sexualité juvénile, au-delà du fait qu'il particularise son œuvre et notamment son cinéma comme étant celui de la fin de l'innocence, constitue un acte iconoclaste contre la mythologie américaine et se traduit visuellement par des partis pris esthétiques dérégulés - dé-réglés ${ }^{13}$ (monstration frontale de la nudité, gros plan caressant, surexposition de l'inconvenant, lumière sale, cadrage chirurgical, image obstruée). Si le refus des règles et la liberté de transgresser caractérisent l'ipséité de la posture clarkienne, le spectateur, acculé à un face à face avec des images qu'il ne sait pas voir, n'en demeure pas pour autant émancipé. Désérotisée et désérotisante, la mise en scène de la jouissance est construite de sorte que l'engagement potentiel de ce dernier dans l'image soit d'emblée endigué par une impossibilité de la désirer.

8 Cette façon de briser l'éros, de profaner l'image de la sexualité, de la rendre repoussante (image-répulsion, spectacle-punition) s'élabore à partir de ce que l'on se propose de nommer la signifiance du détail. Excès de visibilité ou d'audibilité, le détail chez Clark s'appréhende à la faveur d'un étirement du temps que seule une caméra tactile et intrusive peut impressionner. Un étirement, qui s'éprouve au moyen d'une durée malléable - dont on sait depuis Bergson à quel point elle est intérieure, intime et déspatialisée ${ }^{14}$ - et qui paradoxalement se construit sur une fragmentation de l'instant (sa linéarité est scindée). Néanmoins, cette division du représenté s'effectue de sorte que le passage d'un 
plan à un autre, non seulement ne suspend pas la permanence de l'interaction sexuelle, le montage s'oublie, mais il en assure le prolongement en insistant, par l'ajout de plans dérangeants (vision rapprochée, points de vue scopiques, caméra participante), sur la continuité de la performance. Et ce procédé, "être collé au plus près», a pour conséquences, d'une part, de créer un effet de longue durée, une suite interminable que l'on voudrait passer, ne pas voir, et d'autre part, de placer la performance au participe présent ${ }^{15}$ en provoquant un sentiment d'immédiateté et de proximité.

C'est donc par l'entremise de ce temps étiré que le détail va se signifier. Incisif et cuisant, il est cet élément rendu présent, prégnant et irritant, à l'instar du «troisième sens » de Barthes ou plutôt de son punctum en photographie ${ }^{16}$, qui excède l'image et point le spectateur en l'apostrophant désagréablement. In concreto, ce sont les bruits de succion dans le prologue libidinal de Kids, perceptibles avant l'image, qui persistent péniblement tout le long de la séquence, la rendant encore plus dérangeante ${ }^{17}$ - on les retrouve quasi systématiquement dans les scènes de luxure (de tous les films). Ce sont également les bruits de claquement des sexes en action, à peine audibles mais qui parasitent l'ouie tout en appuyant le visible. Ce sont aussi, les cris jouissifs, insupportables, qui émanent de la vidéo porno alors que la caméra filme le viol d'Ali (Bully). De même dans Kids, on pense au crissement du canapé qui supporte Jennie en train d'être violée. Et visuellement, à l'intérieur du cadre, littéralement dans l'image indépendamment de la bande-sonore, on s'affecte de voir :

[...] les socquettes blanches de Jenny [sic] (Chloë Sevigny) quand Casper abuse d'elle sur le canapé; des socquettes qui focalisent le regard et résument à elles seules toute la scène ; des socquettes insupportables, inoubliables. ${ }^{18}$

Ou pareillement, la chemise rouge de Bobby, au moment de la scène du meurtre avorté, lors de son rapport sexuel à peine consenti avec Ali (il est précédé du viol et n'advient que pour accomplir le crime). Une chemise que l'on souhaiterait ne plus avoir à regarder, à subir - notons ici l'adresse de Clark pour accentuer le malaise du spectateur : en dilatant le temps, il contraint ce dernier à vouloir l'accélérer, or, vouloir accélérer le temps c'est vouloir passer au meurtre, et donc le légitimer ${ }^{19}$. Et c'est encore, le filet de bave sur la bouche de Tate (Ken Park), en pleine masturbation, dont l'écho visuel (la giclée de sperme) viendra viscéralement imprimer l'image et la maculer d'un second rejet, celui du spectateur.

11 À travers ces éléments supplétifs et poignants, Clark génère donc une image indésirable et impossible à convoiter. Lestée d'une culpabilité dont la charge travaille à corrompre les corps, cette image coupable, virale, impose son mal au regard et contribue à intensifier le rejet, élément principiel à bien des égards de son cinéma, par lequel tout se passe: impossibilité de transmission sinon par contagion dans la diégèse, refus de dissimuler le visible et le dicible sinon par exagération/saturation dans le plan et incapacité d'investissement dans la fiction sinon par obligation du regard spectatoriel.

\section{Toucher le fond}

Du trash au clash en image, la perception est insolite. Imagerie sexuelle et images sexuées ponctuent un système de représentation organique dans lequel l'exposition des sexes se mêle à la figuration de l'ordinaire. Incorporée à une structure narrative de facture classique, l'iconographie ob-scène s'emplit d'une charge dissensuelle et devient cette 
image tactile qui déborde la visibilité en se dérobant de la suprématie du récit et de l'action.

L'insertion de séquences à caractère sexuel dans la trame de la fiction traditionnelle crée une interruption, une césure dans l'appréhension de la représentation, laquelle, couplée à ses éléments délétères, vient mettre à mal la perception spectatorielle. Cette faille dans la vision, véritable catalyseur disruptif, qui n'est pas sans rappeler l'esthétique du choc théorisée par Walter Benjamin (dont les déterminations marquent un nouveau type de perception qu'il nomme " tactile ») ${ }^{20}$, ouvre un nouveau rapport à l'image dans lequel les habitudes perceptives du spectateur ainsi que son investissement dans la fiction (sa participation affective) sont contrariés. Si l'inclusion de plans luxurieux, dans la continuité « paisible » de la narration, se décharge de l'efficience de la praxis politique du montage $\operatorname{choc}^{21}$ (revendiquée par Benjamin à la suite de la théorie du «montage des attractions " d'Eisenstein) ${ }^{22}$, le cinéma de Clark renouvelle toutefois cette idée selon laquelle la fracture liée à l'esthétique du choc engage une perception « tactile ${ }^{23}$, c'est-àdire une perception disparate, fondée sur la « distraction $»^{24}$ et la diversion. En effet, la rupture, provoquée par le contraste visuel que forme l'exhibition des sexes à l'intérieur de la représentation classique, entrave "l'immersion fictionnelle ${ }^{25} \mathrm{du}$ spectateur, il n'est plus plongé dans l'action du film, et par là même, favorise une réception distraite en venant le heurter. Pour autant, si le cinéma de Clark réactualise la dimension tactile de la perception visuelle, celle-ci n'est en revanche plus à même d'être pensée en opposition à la perception contemplative, "auratique ", de la tradition picturale telle que l'a définie Benjamin. Pervertie, elle diffère de la perception passive, " apathique », du spectateur de film qui, accoutumé à certains modes de réception normalisant l'apparition du sexe à l'écran ${ }^{26}$, ne saurait plus voir.

En d'autres termes, la dé-monstration de Clark, fondée sur une exagération thématique et formelle de l'intime et du trash, laquelle est soutenue par la surexposition des corps licencieux, vient rompre la passivité du spectateur, qui, placé dans une position ambiguë dont il ne peut se départir, est condamné à devoir consommer l'image. Ainsi, pris en otage, nous sommes donc contraints d'en pénétrer le fond en nous interrogeant sur les modalités de notre propre regard. Sommes-nous, à notre tour, coupables de ne pas voir? De trop voir? De mal regarder? Ou bien la culpabilité revient-elle seulement à l'image : trahison, dissimulation, exhibition? Telle est la réflexivité (la singularité) du regard clarkien. Un regard décalé qui accompagne l'œil de la caméra pour mieux s'en défaire. Un regard scrutateur appelé à sonder l'image, la chair, les apparences. Un regard engagé dans une expérience du toucher, en tant qu'il est invité, par le biais d'une esthétique de la proximité, à dépecer l'image pour en saisir la profondeur. Un regard haptique et décadré.

\section{NOTES}

3. Nous nous appuyons sur la définition de Gilles Deleuze, en référence à Aloïs Riegl: «L'haptique, du verbe grec aptô (toucher), ne désigne pas une relation extrinsèque de l'œil au 
toucher, mais une "possibilité du regard", un type de vision distinct de l'optique [...]». (Gilles Deleuze, Francis Bacon. Logique de la sensation, Paris, Éditions du Seuil, 2002, p. 116.)

4. Le regard est amené à fonctionner comme le tact : " [...] on voit de si près qu'on touche ». (Jacques Aumont, De l'esthétique au présent, Paris, De Boeck Université, 1998, p. 21). Voir également Anna Muslewski, «La chair de l'image. L'expérience optico-tactile dans le cinéma de Larry Clark », in Cadrage, Avril 2010.

5. Mia Hansen-Love, « Portraits crachés », Cahiers du cinéma, n 583, Octobre 2003, p. 23.

6. Ils assassinent leur camarade Bobby, le bully.

7. Le film propose une lecture métaphorique de l'affranchissement de la «loi-du-Père » que l'on peut étendre à l'ensemble de l'œuvre clarkien.

8. Remake du film homonyme de Roger Corman sorti en 1958, Teenage Caveman est le seul téléfilm dans la filmographie de Clark.

9. Shawn couche avec la mère de sa petite amie, Peaches est contrainte d'épouser son géniteur et Claude est abusé par son père durant son sommeil.

10. Et c'est bien là toute la question du cinéma de Clark, est-il possible d'approcher l'intimité du monde des teens sans tomber dans l'exploitation que l'on dénonce?

11. Terme emprunté au vocabulaire de la psychologie qui qualifie: « les conduites par lesquelles un sujet cherche à satisfaire des besoins personnels, par exemple à être aimé d'un partenaire sexuel et à disposer de lui sans conditions ». (Henri Piéron, Vocabulaire de la psychologie, Paris, PUF, « Quadrige », 2003, 4 éditions, p. 63.)

12. En tant que déchirure, elle modifie l'état du corps.

13. Pour Larry Clark, il n'y a pas de règles, ni en cinéma, ni en photographie : « Fuck rules, there are no rules for me, I never thing in those terms. » (Larry Clark, « Larry Clark par Larry Clark : une leçon de cinéma ", Conférence de la Cinémathèque Française, 09 Octobre 2010, [En ligne], site web Canal-U, http://www.canal-u.tv).

14. Henri Bergson, Essais sur les données immédiates de la conscience, Paris, PUF, « Quadrige », 2007, $9^{e}$ éd., et La pensée et le mouvant, Paris, PUF, « Quadrige ", 2009, 16 éd.

15. À l'instar de la forme étymologique du mot adolescent comme l'a justement rappelé Patrice Blouin. (Patrice Blouin. et al., "L'Amérique au risque du participe présent », Cahiers du cinéma, op.cit., p. 20.)

16. Le sens obtus caractérise : « une émotion qui désigne simplement ce qu'on aime, ce qu'on veut défendre ; c'est une émotion-valeur, une évaluation» (p. 493), ce qui n'est pas le cas du détail clarkien qui renvoie davantage à un sentiment désagréable, relatif à cette " piqûre » dont parle Barthes pour qualifier le punctum. Si le détail clarkien relève du «troisième sens » c'est essentiellement en raison de sa nature d'excédent qui interpelle le spectateur au-delà du récit et de la représentation. (Roland Barthes, "Le troisième sens " in CEuvres complètes, Tome 3, Paris, Seuil, 2002, pp. 485-506 et La Chambre claire, Paris, Éditions de l'Étoile, Gallimard, Le Seuil, 1980, p.

49.)

17. La séquence présente une scène de sexe entre deux (très) jeunes adolescents.

18. Iannis Katsahnias, «Qu'est-ce qui s'est passé ? », Cahiers du cinéma, n498, Janvier 1996, p. 45. Nous soulignons.

19. Le spectateur de Bully n'est effectivement jamais épargné. Déstabilisé par un léger sentiment de légitimité (à propos du meurtre) qu'une réprobation morale ne parvient pas totalement à effacer, il doit sans cesse faire face à la culpabilité d'une position ambiguë (légitimer ou condamner?).

20. Dans «L'CEuvre d'art à l'époque de la reproductibilité technique ", Walter Benjamin examine les mutations de l'art engendrées par le développement de la technique et réfléchit à la manière dont les nouveaux media (le cinéma et la photographie) bouleversent la perception sensible. Au regard des œuvres dadaïstes, lesquelles, composées d'éléments hétérogènes, développent une esthétique du choc qui modifie la perception artistique (contemplative), il analyse la nouvelle 
modalité du rapport perceptif instaurée par l'image cinématographique. En détruisant l'aura de l'œuvre et en entravant la contemplation esthétique, l'effet choc du montage (au cinéma et dans les poèmes et peintures dadaïstes), crée un renversement des habitudes perceptives du spectateur et de fait, engendre une autre expérience esthétique qui, non seulement abolit la fonction artistique de l'image, elle n'est plus rituelle, mais en outre, marque l'émergence de sa nouvelle fonction, elle devient politique. L'esthétique du choc incite donc à une prise de conscience politique et ouvre ce nouveau rapport perceptif que Walter Benjamin appelle tactile. (Walter Benjamin, "L'CEuvre d'art à l'époque de sa reproductibilité technique » in Euvres III, Paris, Gallimard, 2000, pp. 267-316.)

21. Clark ne cherche pas à façonner un nouveau spectateur qui serait capable de réagir politiquement. De plus, il va de soi que le contexte auquel se réfère Walter Benjamin, le trauma de la Première Guerre mondiale, n'a plus rien à voir avec celui des films clarkiens.

22. Jean-Philippe Uzel a montré comment Walter Benjamin, à partir des analyses d'Eisenstein sur le «montage des attractions", a envisagé le choc du montage comme un catalyseur de l'action politique. (Jean-Philippe Uzel, «Le montage : de la vision à l'action ", CiNéMAS, Les dispositifs de médiation au cinéma, vol. 9, n¹, Montréal, 1998, pp. 63-78.)

23. Walter Benjamin, «L'ÆEuvre d'art à l'époque de sa reproductibilité technique ", op.cit., p. 309.

24. Walter Benjamin, ibid., pp. 308-313.

25. Jean-Marie Schaeffer, Pourquoi la fiction?, Paris, Éditions du Seuil, 1999, p 133.

26. Ce sera le sujet d'Impaled (2006). À force d'exploitation et de répétitions récurrentes dans le secteur de l'entertainment (et ailleurs), le spectateur est devenu indifférent à la représentation du sexe. C'est pourquoi, il est désormais opportun de se demander si : « Le cinéma porno fait [...] encore bander? » - formule qui résume à elle seule l'idée d'amenuisement voire de dissolution de la charge subversive de la pornographie, qu'Impaled développera. (Jean-Baptiste Thoret et Stéphane Bou, « Le cinéma porno fait-il encore bander? » émission radiophonique, « Pendant les travaux le cinéma reste ouvert », France Inter, Août 2012).

1. Kids (1995), Another Day in Paradise (1999), Bully (2001), Teenage Caveman (2002), Ken Park (2003), Wassup Rockers (2006) et Impaled (2012).

2. Teenage Lust (1983), Larry Clark 1992 (1992), The Perfect Childhood (1993), punkPicasso (2003) et Larry Clark Los Angeles 2003-2006 (2007).

\section{RÉSUMÉS}

Larry Clark est un cinéaste du corps et du tact. De Tulsa (1971), son premier album photographique, à Marfa Girl (2012) son dernier film en date, en passant par l'ensemble de sa filmographie ${ }^{1}$ et de ses recueils de photographies ${ }^{2}$, il ne cesse de traquer les corps juvéniles de l'adolescence américaine marginale, d'en scruter la chair délabrée et d'en sonder les dérives, le mal-être. Son œuvre, art du contact et de la palpation, engage une relation particulière au toucher dans laquelle le regard (des personnages, du spectateur et du cinéaste) se fait préhensif et devient haptique. Cet article a pour dessein d'explorer la dimension tactile du cinéma clarkien et d'en analyser les modalités sensibles en essayant de montrer comment l'image chez Clark, que l'on dépeint souvent comme perverse et voyeuriste, est avant tout une image réflexive.

The Guilty-Image : Haptic Look in Larry Clark's Cinema 
Larry Clark is a filmmaker of body and touch. From his first photograph album Tulsa (1971) to Marfa Girl (2012) his latest film - and in between his entire filmography ${ }^{1}$ and collection of photographs $^{2}$ - he has relentlessly tracked down the youthful bodies of American fringe adolescence, examined their damaged flesh and probed drifts and malaise. His work, as an art of touch and palpation, creates a special relation to the sense of touch in which the characters', spectators' and film director's look becomes prehensive and haptical. The present article aims to explore the tactile dimension of Clark's cinema and analyze the sensitive modalities by attempting to show how the picture in Clark's works, which is often depicted as perverted and voyeurist, is above all a reflexive image.

\section{AUTEUR}

\section{ANNA MUSLEWSKI}

Anna Muslewski, doctorante en études cinématographiques et audiovisuelles sous la direction de Giusy Pisano, prépare une thèse sur le cinéma de Larry Clark. Elle travaille notamment sur les relations qu'entretiennent la photographie et le cinéma ainsi que sur la représentation iconographique du corps dans son œuvre. 